\title{
Therapeutic Equivalency Study
}

National Cancer Institute

\section{Source}

National Cancer Institute. Therapeutic Equivalency Study. NCI Thesaurus. Code C49665.

A study most often used to compare the efficacy of different formulations to treat a given disease. It is the testing of an old versus a new formulation in healthy volunteers or subjects with the disease under study and usually in one dose. 